\title{
Reperfusion pulmonary edema in children with tetralogy of Fallot, pulmonary atresia, and major aortopulmonary collateral arteries undergoing unifocalization procedures: A pilot study examining potential pathophysiologic mechanisms and clinical significance
}

\author{
Ritu Asija, MD, ${ }^{a}$ Stephen J. Roth, MD, MPH, ${ }^{a}$ Frank L. Hanley, MD, ${ }^{\mathrm{b}}$ Lynn Peng, MD, ${ }^{\mathrm{a}}$ Kathleen Liu, MD, \\ Jason Abbott, BA, ${ }^{\mathrm{c}}$ Hanjing Zhuo, MD, MPH, ${ }^{\mathrm{c}}$ and Michael Matthay, $\mathrm{MD}^{\mathrm{c}}$
}

\begin{abstract}
Objective: Children with tetralogy of Fallot (TOF), pulmonary atresia (PA), and major aortopulmonary collateral arteries (MAPCAs) are at risk for reperfusion pulmonary edema (RPE) after unifocalization procedures to reconstruct the central pulmonary arteries. The purpose of this study was to determine the incidence of RPE, describe the clinical course of patients with RPE, and explore the mechanism of RPE in this population by measuring plasma biomarkers of alveolar epithelial and endothelial injury and lung inflammation.
\end{abstract}

\begin{abstract}
Methods: Levels of plasma receptor for advanced glycation end products (RAGE), intercellular adhesion molecule 1 (ICAM-1), and interleukin 6 (IL-6) were measured at baseline and postoperative day (POD) 0, 1, and 2 after unifocalization. A pediatric radiologist reviewed chest radiographs from the same time points and scored each lung segment for the degree of pulmonary edema. A pediatric interventional cardiologist reviewed the preoperative angiograms for each patient and determined the degree of stenosis for each aortopulmonary collateral vessel. RPE was defined as localized pulmonary edema with a pulmonary edema score of at least 2 occurring in the lung segment demonstrating the greatest degree of angiographic stenosis within the first 48 hours after surgery and with resolution by discharge.

Results: Thirty-five patients who underwent 37 unifocalization procedures were enrolled, and 32 patients were included in the analysis. Of these, 16 of $32(50 \%)$ demonstrated evidence of RPE based on our defined criteria. There was no significant difference in RAGE $(P=.60)$, ICAM-1 $(P=.34)$, or IL-6 $(P=.31)$ levels between those with and without RPE at any time point. The mean duration of mechanical ventilation in patients with RPE versus those without was not significantly different ( $5.1 \pm 4.2$ vs $5.6 \pm 4.5$ days, respectively; $P=.57$ ).

Conclusions: Fifty percent of children with TOF/PA/MAPCAs undergoing unifocalization surgery developed RPE. Levels of plasma biomarkers of alveolar epithelial and endothelial injury and lung inflammation were not increased in patients with RPE compared with those without RPE. The presence of RPE did not affect the duration of respiratory failure and mechanical ventilation. The process of RPE is clinically self-limited and seems unlikely to be associated with vascular changes. (J Thorac Cardiovasc Surg 2014;148:1560-5)
\end{abstract}

Tetralogy of Fallot (TOF), pulmonary atresia (PA), and major aortopulmonary collateral arteries (MAPCAs) is a form of cyanotic congenital heart disease in which children are born with TOF plus underdeveloped or absent central pulmonary arteries. Aortopulmonary collaterals provide the

From the Division of Pediatric Cardiology, ${ }^{\text {a }}$ Department of Pediatrics, Division of Pediatric Cardiothoracic Surgery, ${ }^{\mathrm{b}}$ Department of Cardiothoracic Surgery, Stanford University School of Medicine, Palo Alto, Calif; and The Cardiovascular Research Institute, ${ }^{\mathrm{c}}$ Departments of Medicine and Anesthesia, University of California San Francisco, San Francisco, Calif.

Disclosures: Kathleen Liu reports consulting fees from Astute, Abbvie, Complexa, and Cytopherx, and equity ownership in Amgen. Michael Matthay reports consulting fees from Cerus Inc, Roche-Genentec, and GlaxoSmithKline, as well as grant support from GlaxoSmithKline. All other authors have nothing to disclose with regard to commercial support.

Received for publication Oct 28, 2013; revisions received Dec 31, 2013; accepted for publication Jan 20, 2014; available ahead of print Feb 16, 2014.

Address for reprints: Ritu Asija, MD, 750 Welch Rd, Suite 305, Palo Alto, CA 94304 (E-mail: rasija@stanford.edu).

0022-5223/\$36.00

Copyright (c) 2014 by The American Association for Thoracic Surgery

http://dx.doi.org/10.1016/j.jtcvs.2014.01.017 only source of pulmonary blood flow, resulting in heterogeneous flow to different lung segments. ${ }^{1}$ Surgical repair requires the establishment of controlled blood flow into all lung segments by unifocalizing collateral vessels into the native or reconstructed central pulmonary arteries. ${ }^{2}$ Given the propensity for MAPCAs to have highly variable anatomic courses and degrees of stenosis, extensive reconstruction of the collateral vessels is often required. At our institution, we have developed a surgical approach based on the degree of segmental-level stenosis and the presence of true pulmonary arteries that emphasizes early complete unifocalization and intracardiac repair with ventricular septal defect (VSD) closure and placement of a right ventricle-to-pulmonary artery conduit. ${ }^{3,4}$

We recently showed that patients undergoing unifocalization surgery are at risk for the development of reperfusion pulmonary edema (RPE), and that the degree of preoperative angiographic stenosis in MAPCAs was a significant predictor for the development and severity of RPE. ${ }^{5} \mathrm{RPE}$ 


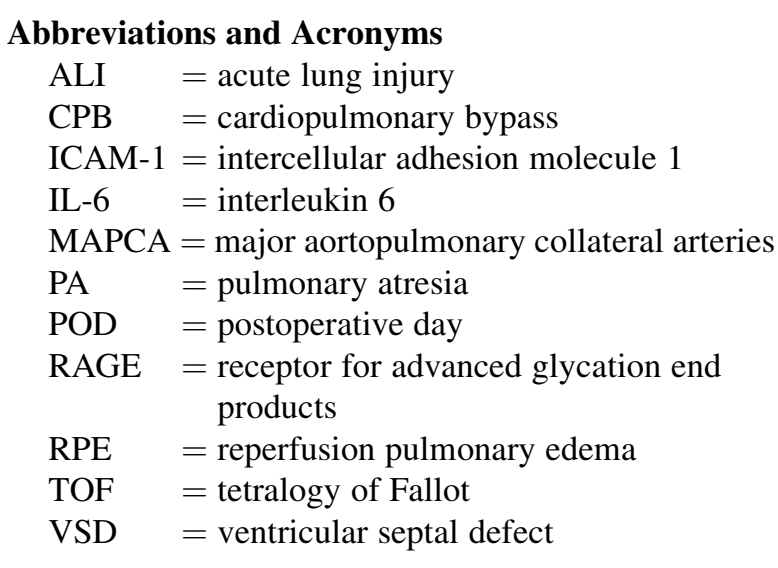

has been studied in other contexts as well. Lung injury from cardiopulmonary bypass is believed to be related to ischemia-reperfusion mechanisms and can have important clinical implications for infants undergoing congenital heart surgery. ${ }^{6,7}$ RPE has also been reported in children with branch pulmonary artery stenosis and selected congenital heart defects after pulmonary artery balloon dilation., ${ }^{8,9}$ We hypothesized that children with TOF/PA/MAPCAs undergoing unifocalization surgery may manifest biological evidence of acute lung injury (ALI) similar to adult patients who develop RPE after lung transplant, and we therefore chose to study 3 biomarkers known to have pathophysiologic and prognostic value in adults with ALI. One such molecule is receptor for advanced glycation end products (RAGE), a marker of alveolar type I epithelial cellular injury. ${ }^{10}$ RAGE has been shown to correlate with the severity of primary graft dysfunction in adults undergoing lung transplantation. Increased levels of plasma RAGE are a marker of alveolar type I epithelial cell injury and impaired alveolar fluid clearance, and they correlate with the severity of primary graft dysfunction in adults undergoing lung transplant. ${ }^{11,12}$ Intercellular adhesion molecule 1 (ICAM-1) is a marker of alveolar epithelial and endothelial injury and alveolar macrophage activation. ${ }^{13}$ In children with ALI, ICAM-1 levels early in the course correlate with the duration of mechanical ventilation and survival. ${ }^{14}$ Interleukin-6 (IL-6) is a marker of lung inflammation that has been shown to correlate with the degree of pulmonary edema after lung transplant. ${ }^{15}$ The primary goals of our study were to describe the incidence and clinical course of RPE in patients with TOF/PA/MAPCAs after unifocalization surgery and to better understand its pathophysiology by assessing plasma markers of alveolar epithelial and endothelial injury and lung inflammation.

\section{METHODS}

All patients with TOF/PA/MAPCAs presenting for unifocalization or pulmonary artery revision procedures were eligible for enrollment in this prospective study, including those with additional structural cardiac abnormalities requiring surgical intervention. Patients with single ventricle cardiac anatomy, preoperative respiratory failure, or known infection were excluded. The enrollment period was from May, 2009, to January, 2011.

An experienced pediatric radiologist blinded to the patient's clinical course reviewed a series of chest radiographs for each patient. The radiologist interpreted preoperative, immediate postoperative, postoperative day (POD) 1, and POD 2 radiographs, and scored each for localized pulmonary edema using a scoring system adapted from a previous study assessing pulmonary reperfusion injury in lung transplant recipients. ${ }^{5,16}$ The right upper lobe, right middle lobe, right lower lobe, left upper lobe, lingula, and left lower lobe were all scored on a scale from 0 to 3: 0 for normal lung, 1 for minimal opacity not obscuring lung vessels, 2 for opacity partially obscuring lung vessels, and 3 for opacity completely obscuring lung vessels. Lung vascularity and the presence or absence of atelectasis were also recorded. The suitability of the chest radiograph for inclusion in the study was at the discretion of the radiologist, and the radiograph was to be excluded from analysis if the radiologist could not differentiate pulmonary edema from atelectasis.

The degree of stenosis of each MAPCA was determined by an experienced pediatric interventional cardiologist blinded to the patient's postoperative course and was based on a published protocol from our institution. ${ }^{5}$ Briefly, the width of the narrowest segment of each MAPCA and the widest downstream segment were measured in millimeters. If a MAPCA branched into multiple segments before the largest downstream segment, the total width of all the downstream segments was used. The stenosis was then reported as a ratio of the width of the narrowest segment to the width of the largest segment downstream. The lung segment with the smallest ratio was considered to be the most at risk for the development of RPE.

For purposes of this study, RPE was defined as localized pulmonary edema with a score of at least 2 occurring in the lung segment with the greatest degree of angiographic stenosis within the first 48 hours after surgery and resolving by hospital discharge. Patients with a pulmonary opacity accompanied by fever and increased levels of inflammatory markers were considered to have pneumonia and were excluded from the analysis. Patients were also excluded for pulmonary hemorrhage, defined as the presence of persistent frankly bloody tracheal secretions in the first 48 hours postoperatively.

Blood samples were collected from each patient's indwelling arterial catheter at 4 time points: preoperative, immediate postoperative, and on POD 1 and 2. The blood was collected in EDTA-treated tubes and centrifuged for 10 minutes at $3000 \mathrm{~g}$. Plasma samples were then aliquoted and stored at $-80^{\circ} \mathrm{C}$. Using these samples, the levels of RAGE, ICAM-1, and IL-6 were measured using commercially available enzyme-linked immunosorbent assays (R\&D Systems, Minneapolis, Minn).

Partial pressure of oxygen to fractional inspired oxygen $(\mathrm{P} / \mathrm{F})$ ratios and alveolar to arterial oxygen gradients $\left(\mathrm{A}-\mathrm{a} \mathrm{O}_{2}\right)$ were calculated daily on POD 0,1 , and 2 for patients undergoing complete intracardiac repair with VSD closure and no residual intracardiac shunt. The $\mathrm{A}-\mathrm{a} \mathrm{O}_{2}$ gradient was calculated according to the following equation: $\left(713 \times \mathrm{FiO}_{2}-\mathrm{PaCO}_{2} / 0.8\right)-\mathrm{PaO}_{2}$ where $\mathrm{FiO}_{2}$ is the fractional inspired oxygen, $\mathrm{PaCO}_{2}$ is the arterial carbon dioxide tension, and $\mathrm{PaO}_{2}$ is the arterial oxygen tension. The highest $\mathrm{P} / \mathrm{F}$ values and lowest $\mathrm{A}-\mathrm{a} \mathrm{O}_{2}$ gradient measured in the first 48 hours postoperatively were compared between patients with RPE and those without.

Analysis of covariance was used to analyze the impact of RPE on the biomarker levels at the postoperative, POD 1, and POD 2 time points controlling for the baseline level. A generalized estimating equation approach was used to test the impact of RPE on RAGE, ICAM-1, and IL-6, taking repeated measures into account and using an exchangeable correlation matrix. The Student $t$ test was used to analyze angiographic stenosis and clinical data. All statistical analyses were performed using commercial software (STATA 12.0, College Station, Tex). The study was approved by the Institutional Review Boards of Stanford University and the University of California, San Francisco. 


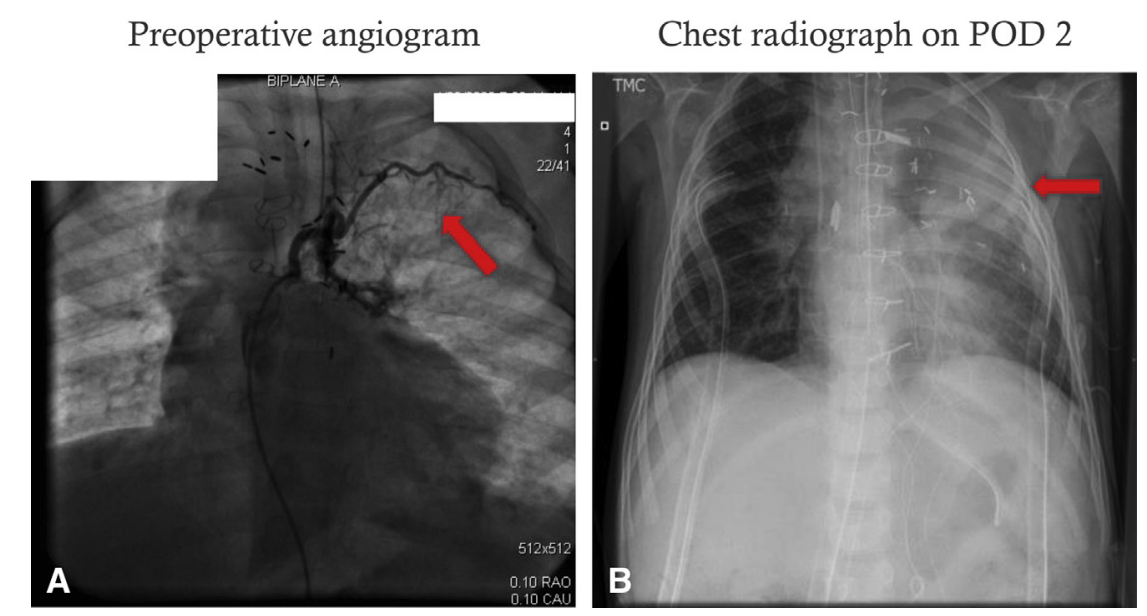

FIGURE 1. Example of long-segment stenosis in the left upper lobe (arrow in A) and subsequent reperfusion pulmonary edema in this area (arrow in B). $P O D$, Postoperative day.

\section{RESULTS \\ Demographics}

Thirty-five consecutive patients undergoing 37 procedures were enrolled during the study period. Five patients were excluded; 1 patient developed postoperative pneumonia and 4 patients had their angiograms performed at other institutions and the images did not provide adequate anatomic definition. No patients were excluded because of suboptimal chest radiographs. There were 16 boys and 16 girls. The median age was 11.5 months (range, 3-132 months) and the median weight was $7.3 \mathrm{~kg}$ (range, $4.5-31.2 \mathrm{~kg}$ ).

\section{Intraoperative Course}

The 32 patients included in the analysis underwent 1 of the following 4 types of surgery: complete, single-stage unifocalization with intracardiac repair $(\mathrm{n}=12)$, intracardiac repair as part of a staged unifocalization approach $(\mathrm{n}=11)$, partial unifocalization and aortopulmonary shunt placement without intracardiac repair $(\mathrm{n}=7)$, and unifocalization revision or pulmonary arterioplasty and right ventricle-to-pulmonary artery conduit replacement $(\mathrm{n}=2)$. Two patients undergoing partial unifocalization procedures did not require cardiopulmonary bypass (CPB), and both met the criteria for RPE. In the remaining patients who did require $\mathrm{CPB}$, there was no significant difference in the mean CPB time between patients with RPE $(271 \pm 146 \mathrm{mi}-$ nutes) compared with those without RPE ( $274 \pm 57$ minutes $)$ $(P=.07)$. All patients had good biventricular systolic function based on qualitative interpretation of postoperative echocardiograms, and there were no significant residual lesions (VSDs, right ventricular-to-pulmonary artery conduit stenosis or insufficiency, aortopulmonary shunt stenosis, or occlusion). No patients developed clinically detectable postoperative pulmonary hemorrhage.

\section{Criteria for RPE}

Review of the chest radiographs for the 32 patients included showed that 16 patients $(50 \%)$ met the criteria for RPE (a pulmonary edema score of 2 or greater within the first 48 hours postoperatively with resolution by discharge in the lobe of the most significant angiographic stenosis). Of the 16 patients who did not meet the criteria, 7 had a pulmonary edema score greater than 2 in at least 1 lung segment, but the edema was not localized to the lung segment with the most significant angiographic stenosis. The remaining 9 patients did not demonstrate any pulmonary edema and essentially had normal chest radiographs. No chest radiographs were excluded from the analysis.

Amongst the 7 patients with aortopulmonary shunts as their sole source of pulmonary blood flow, 3 developed RPE (43\%). The remaining 25 patients had a septated circulation with a right ventricle-to-pulmonary artery conduit as their source of pulmonary blood flow, and 13 patients (52\%) developed RPE.

The mean ratio of the narrowest-to-widest segment in the smallest collateral vessels measured on an angiogram in the RPE group was $0.38 \pm 0.16$, whereas this ratio was $0.31 \pm$ $0.09(P=.07)$ in those without RPE. An example of RPE in 1 patient is shown in Figure 1. It demonstrates the development of RPE in the left upper lobe lung segment supplied by a significantly narrowed collateral vessel identified on the preoperative angiogram. The stenosis-to-downstream ratio for this collateral vessel was 0.28 .

\section{Biomarker Results}

Complete biomarker data were available for 8 patients without RPE and 9 patients with RPE. There was insufficient plasma volume for analysis of all 3 biomarkers in the remaining patients. There was no significant difference in RAGE, ICAM-1, and IL-6 levels in patients with and without RPE at any time point (Figure 2, $A-C$ ). Taking 

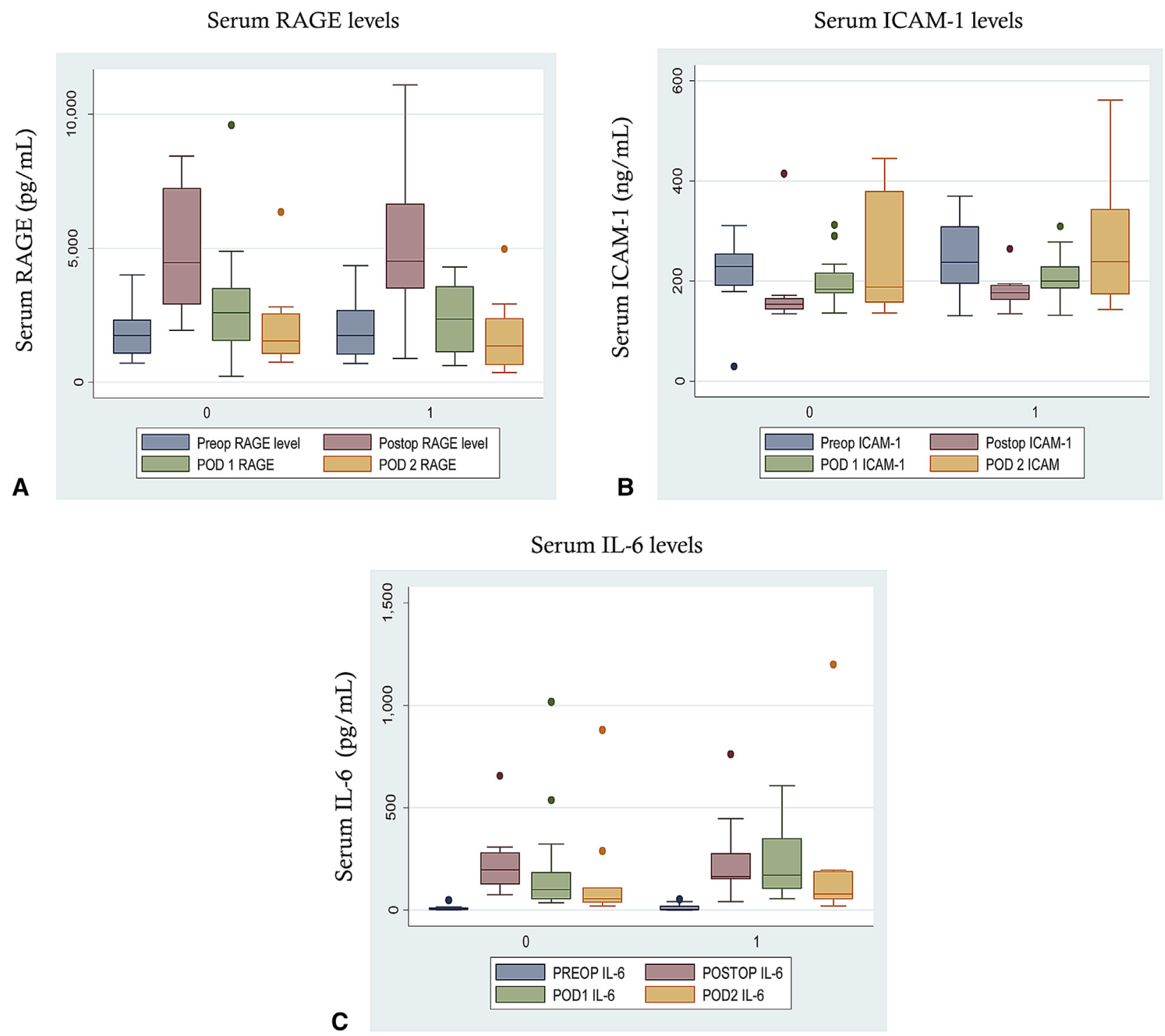

FIGURE 2. Box-and-whisker plots comparing biomarker levels at 4 time points in patients without (0) and with (1) reperfusion pulmonary edema. (Error bars represent maximum and minimum values excluding outliers, represented by outside dots. Columns depict 25 th percentile, median, and 75 th percentile values). A, Serum RAGE levels. B, Serum ICAM-1 levels. C, Serum IL-6 levels. Single outlier value of 4163 pg/mL on POD 2 in reperfusion pulmonary edema group excluded to improve graph visualization. RAGE, Receptor for advanced glycation end products; Preop, preoperative; Postop, postoperative; $P O D$, postoperative day; ICAM-1, intercellular adhesion molecule $1 ; I L-6$, interleukin 6.

repeated measures into account and controlling for baseline preoperative levels, there was no significant difference in the levels of RAGE $(P=.60)$, ICAM-1 $(P=.34)$, or IL-6 $(P=.31)$. Levels of RAGE and IL-6 tended to increase in the immediate postoperative period and then decrease on POD 1 and 2 in both groups, whereas ICAM-1 levels were lower than baseline immediately after surgery and increased back toward baseline on POD 1 and 2 .

\section{Clinical Results}

The mean duration of mechanical ventilation in patients with RPE versus those without was not significantly different $(5.1 \pm 4.2$ vs $5.6 \pm 4.5$ days, respectively; $P=.57)$. Twenty-five patients underwent complete intracardiac repair or unifocalization revision procedures and did not have residual intracardiac shunts, and $13(52 \%)$ demonstrated evidence of RPE. The average P/F ratio of patients with RPE in this group was $216 \pm 75$, and 241 \pm 81 for those without RPE was $(P=.7)$. The average A-a $\mathrm{O}_{2}$ for those with $\mathrm{RPE}$ was $206 \pm 81 \mathrm{~mm} \mathrm{Hg}$ and $219 \pm 73 \mathrm{~mm} \mathrm{Hg}$ for those without $(P=.9)$. Of the $7 \mathrm{pa}-$ tients who underwent partial unifocalization with pulmonary blood flow supplied by an aortopulmonary shunt, 3 developed RPE. 


\section{DISCUSSION}

Although RPE has been described in the literature, little is known about its pathophysiology and clinical impact in children with TOF/PA/MAPCAs undergoing unifocalization procedures. ${ }^{5,8}$ To our knowledge, this is the first study designed with the explicit goal of understanding the pathophysiology of RPE. Using plasma markers of lung injury that have pathophysiologic and prognostic significance in adults and children with ALI and pulmonary edema after lung transplant, we did not detect a difference in patients with radiographic evidence of lung injury compared with those without in our study population.

One possible explanation is that the extent and severity of pulmonary edema are different in patients with RPE after unifocalization procedures compared with patients with ALI and posttransplant pulmonary edema. Patients with RPE in our population may not develop severe enough alveolar endothelial or epithelial injury and lung inflammation to produce a difference in these biological markers. The definition of ALI includes the presence of bilateral infiltrates on a chest radiograph, indicating that the lungs are diffusely affected. ${ }^{17}$ In our patient population, RPE was defined as focal pulmonary edema occurring in a lung region associated with vascular obstruction on angiography. By definition, the degree of lung injury is limited to specific lung segments. Levels of RAGE, ICAM-1, and IL-6 may not have been significantly different between the 2 groups because the degree of pulmonary edema was not extensive enough to cause an important difference in protein expression.

Another potential explanation is that the mechanism by which pulmonary edema develops may be different in our population than in patients with ALI. The pathophysiology of ALI has been found to be related to capillary injury with resultant capillary leak and the development of proteinaceous pulmonary edema. ${ }^{18-20}$ Although the possibility of capillary injury cannot be excluded, our patients may have developed hydrostatic edema as a consequence of overperfusion in a previously restricted vascular bed. Patients with significant narrowing of their MAPCAs have decreased blood flow and pressure in the pulmonary microvasculature beyond the narrowings. After unifocalization surgery, blood flow increases through the previously stenotic vessels resulting in increased pressure. The microvasculature cannot immediately restrict flow, and therefore hydrostatic pulmonary edema develops. The pathogenesis of such overperfusion hydrostatic edema was first described by Landolt and colleagues ${ }^{21}$ in sheep after pneumonectomy. The lymph-to-plasma ratio in pleural fluid measured from these study sheep was unchanged after increasing pressure and flow to the remaining lung, indicating that capillary leak did not occur.

Hydrostatic pulmonary edema is less clinically severe than edema secondary to lung capillary injury. Hydrostatic pulmonary edema as a pathophysiologic explanation is therefore plausible, given that there was no difference in clinical severity between the 2 groups. There was no difference in the duration of mechanical ventilation between patients with and without RPE, and both groups demonstrated resolution of their pulmonary edema by discharge. In addition, measures of oxygenation, the $\mathrm{P} / \mathrm{F}$ ratio and $\mathrm{A}-\mathrm{a} \mathrm{O}_{2}$ gradient, were similar in patients with and without RPE, indicating that the severity of lung disease may not be clinically significant. The subgroup of our patients undergoing partial unifocalization surgery had surgically placed aortopulmonary shunts to provide pulmonary blood flow and stimulate growth of the pulmonary vasculature before complete intracardiac repair. The pulmonary vascular bed is supplied with systemic-level pressure and flow, and therefore it may be at higher risk for developing hydrostatic pulmonary edema, particularly in segments with low flow preoperatively due to significant vascular restriction. Although only 3 of our patients with this physiology developed RPE, our current pilot study is not powered to detect a difference. Given that patients with pulmonary blood flow via the right ventricle-pulmonary artery conduit developed RPE, it is plausible that the pathophysiology is multifactorial and not related to the pressure of pulmonary blood flow alone. The anatomy, that is, the degree of stenosis, of a vessel before unifocalization may be equally important in determining whether the lung segment develops RPE.

Our study population could be considered at risk for cardiogenic pulmonary edema given that patients were undergoing surgery for congenital heart disease. The pathophysiology of cardiogenic pulmonary edema is different than that of ALI and posttransplant pulmonary edema, and therefore the biological markers of lung injury may also be different. We believe, however, that our study population was at low risk for developing cardiogenic pulmonary edema. Patients with TOF/PA/MAPCAs undergoing unifocalization surgery at our institution are carefully selected for complete intracardiac repair by a well-established surgical algorithm based on low intraoperative pulmonary artery pressures. ${ }^{3}$ Previous work from our institution demonstrated that patients typically have good postoperative cardiopulmonary mechanics after repair of TOF/PA/MAPCAs, and that when they develop prolonged postoperative respiratory failure, it is secondary to pulmonary complications. ${ }^{22}$ In addition, our patients had either qualitatively normal or only mildly decreased systolic biventricular function on postoperative echocardiography. We defined RPE as focal pulmonary edema in the lung region associated with the greatest angiographic stenosis. If the pulmonary edema did not occur in the area of angiographic stenosis, it was not considered to be RPE. Although patients can experience lung inflammation after $\mathrm{CPB}$, there was no difference in CPB times between the 2 groups.

A recent study from our institution demonstrated that the degree of angiographic stenosis on the preoperative 
angiogram correlated with the development of RPE after unifocalization. ${ }^{5}$ Our study also demonstrated the trend that RPE developed in patients with more significant angiographic stenosis, although it did not reach statistical significance, likely secondary to the fact that this was a smaller pilot study.

\section{Limitations}

We believe this is the first study designed to examine possible pathophysiologic mechanisms of RPE in patients with TOF/PA/MAPCAs undergoing unifocalization surgery. One limitation is that no diagnostic criteria exist for RPE in patients with congenital heart disease undergoing procedures to improve or establish pulmonary blood flow, and little is known about its clinical impact. We defined RPE based on our clinical experience and a recent study from our institution, and chest radiographs were scored using a system of grading adapted from lung transplant recipients.

Another limitation is that we had a small number of patients and the study was likely underpowered to detect a difference in the 3 biological markers of lung injury that we chose to measure. We also had a small number of patients with aortopulmonary shunts relative to patients with right ventricle-to-pulmonary artery conduits, limiting our ability to draw conclusions about the impact of systemic-level pressure on the pulmonary microvasculature. Our study was designed to be a pilot for further investigations aimed at understanding the pathophysiology of RPE in children with congenital heart disease.

In our clinical experience, there is a small population of children who experience severe oxygenation deficits after unifocalization surgery that seem to be associated with RPE. These patients have required veno-venous extracorporeal membrane oxygenation to achieve adequate gas exchange, and they typically have complicated clinical courses. We intend to direct our future efforts at these more severe cases to better understand the pathophysiologic mechanisms of RPE in this unique subpopulation of patients with TOF/PA/MAPCAs.

\section{CONCLUSIONS}

Reperfusion pulmonary edema may occur in as many as half of patients with TOF/PA/MAPCAs undergoing unifocalization surgery. Biomarkers of lung injury and the clinical course were not different between children with and without RPE. RPE in our population may be secondary to high-flow, high-pressure hydrostatic pulmonary edema without lung vascular injury. Although we cannot completely exclude the possibility of capillary injury, the process of RPE is clinically self-limited and as such does not seem to involve vascular injury.

\section{References}

1. Hanley FL. MAPCAs, bronchials, monkeys, and men. Eur J Cardiothorac Surg. 2006;29:643-4
2. Reddy VM, Liddicoat JR, Hanley FL. Midline one-stage complete unifocalization and repair of pulmonary atresia with ventricular septal defect and major aortopulmonary collaterals. J Thorac Cardiovasc Surg. 1995;109:832-44; discussion 844-35.

3. Reddy VM, Petrossian E, McElhinney DB, Moore P, Teitel DF, Hanley FL. Onestage complete unifocalization in infants: when should the ventricular septal defect be closed? J Thorac Cardiovasc Surg. 1997;113:858-66; discussion 866-8.

4. Malhotra SP, Hanley FL. Surgical management of pulmonary atresia with ventricular septal defect and major aortopulmonary collaterals: a protocolbased approach. Semin Thorac Cardiovasc Surg Pediatr Card Surg Annu. 2009; 145-51.

5. Maskatia SA, Feinstein JA, Newman B, Hanley FL, Roth SJ. Pulmonary reperfusion injury after the unifocalization procedure for tetralogy of Fallot, pulmonary atresia, and major aortopulmonary collateral arteries. J Thorac Cardiovasc Surg. 2012;144:184-9.

6. Suzuki T, Fukuda T, Ito T, Inoue Y, Cho Y, Kashima I. Continuous pulmonary perfusion during cardiopulmonary bypass prevents lung injury in infants. Ann Thorac Surg. 2000;69:602-6.

7. Qiu W, Zheng L, Gu H, Chen D, Chen Y. Comparison between adult and infant lung injury in a rabbit ischemia-reperfusion model. J Thorac Cardiovasc Surg. 2008;136:352-9.

8. Feinstein JA, Goldhaber SZ, Lock JE, Ferndandes SM, Landzberg MJ. Balloon pulmonary angioplasty for treatment of chronic thromboembolic pulmonary hypertension. Circulation. 2001;103:10-3.

9. Al-Khaldi A, Mohammed Y, Tamimi O, Alharbi A. Early outcomes of total pulmonary arterial reconstruction in patients with arterial tortuosity syndrome. Ann Thorac Surg. 2011;92:698-704; discussion 704.

10. Uchida T, Shirasawa M, Ware LB, Kojima K, Hata Y, Makita K, et al. Receptor for advanced glycation end-products is a marker of type I cell injury in acute lung injury. Am J Respir Crit Care Med. 2006;173:1008-15.

11. Calfee CS, Budev MM, Matthay MA, Church G, Brady S, Uchida T, et al. Plasma receptor for advanced glycation end-products predicts duration of ICU stay and mechanical ventilation in patients after lung transplantation. J Heart Lung Transplant. 2007;26:675-80.

12. Briot R, Frank JA, Uchida T, Lee JW, Calfee CS, Matthay MA. Elevated levels of the receptor for advanced glycation end products, a marker of alveolar epithelial type I cell injury, predict impaired alveolar fluid clearance in isolated perfused human lungs. Chest. 2009;135:269-75.

13. Calfee CS, Eisner MD, Parsons PE, Thompson BT, Conner ER Jr, Matthay MA et al. Soluble intercellular adhesion molecule-1 and clinical outcomes in patients with acute lung injury. Intensive Care Med. 2009;35:248-57.

14. Flori HR, Ware LB, Glidden D, Matthay MA. Early elevation of plasma soluble intercellular adhesion molecule-1 in pediatric acute lung injury identifies patients at increased risk of death and prolonged mechanical ventilation. Pediatr Crit Care Med. 2003;4:315-21.

15. Mathur A, Baz M, Staples ED, Bonnell M, Speckman JM, Hess PJ Jr, et al. Cytokine profile after lung transplantation: correlation with allograft injury. Ann Thorac Surg. 2006;81:1844-9; discussion 1849-50.

16. Anderson DC, Glazer HS, Semenkovich JW, Pilgram TK, Trulock EP, Cooper JD, et al. Lung transplant edema: chest radiography after lung transplantation-the first 10 days. Radiology. 1995;195:275-81.

17. Artigas A, Bernard GR, Carlet J, Dreyfuss D, Gattinoni L, Hudson L, et al. The American-European Consensus Conference on ARDS, part 2: Ventilatory, pharmacologic, supportive therapy, study design strategies, and issues related to recovery and remodeling. Acute respiratory distress syndrome. Am J Respir Crit Care Med. 1998;157(4 Pt 1):1332-47.

18. Pugin J, Verghese G, Widmer MC, Matthay MA. The alveolar space is the site of intense inflammatory and profibrotic reactions in the early phase of acute respiratory distress syndrome. Crit Care Med. 1999;27:304-12.

19. Ware LB, Matthay MA. The acute respiratory distress syndrome. N Engl J Med. 2000;342:1334-49.

20. Matthay MA, Ware LB, Zimmerman GA. The acute respiratory distress syndrome. J Clin Invest. 2012;122:2731-40.

21. Landolt CC, Matthay MA, Albertine KH, Roos PJ, Wiener-Kronish JP, Staub NC. Overperfusion, hypoxia, and increased pressure cause only hydrostatic pulmonary edema in anesthetized sheep. Circ Res. 1983;52: 335-41.

22. Asija R, Hanley FL, Roth SJ. Postoperative respiratory failure in children with tetralogy of Fallot, pulmonary atresia, and major aortopulmonary collaterals: a pilot study. Pediatr Crit Care Med. 2013;14:384-9. 